\author{
Marisa Álvarez Suárez* \\ Javier Domínguez Viera**
}

\title{
LAS DIFERENTES APROXIMACIONES METODOLÓGICAS PARA LA PREVENCIÓN Y DETECCIÓN DE PROBLEMAS REGULATORIOS EN EL ÁMBITO ECONÓMICO
}

La creciente complejidad de las actividades económicas requiere de un esfuerzo de las Administraciones por mejorar la comprensión de la realidad a regular y por evaluar los resultados de su intervención. Para el desarrollo de esta aproximación de buena regulación económica se necesita contar con metodologías de análisis que parten de la Administración, del usuario o del análisis del dato microeconómico. Es necesario continuar con la profundización de estas metodologías y el uso complementario de todas ellas.

\section{The different methodological approaches for the prevention and detection of regulatory issues in the economic sector}

The growing complexity of economic activities requires an effort on the part of Government to improve the understanding of the situation to be regulated and to evaluate the results of its intervention. To develop this approach of sound economic regulation, analytical methodologies based on the perspective of Government, user, or microdata analysis, are needed. It is necessary to continue developing these methodologies and their complementary implementation.

Palabras clave: buena regulación, unidad de mercado, mercado interior, análisis de barreras, libre circulación de bienes y servicios.

Keywords: better regulation, market unity, internal market, barrier analysis, free movement of goods and services.

JEL: H83, L51, O38.

\footnotetext{
* Subdirectora General de Unidad de Mercado, Mejora de la Regulación y Competencia. Ministerio de Economía y Empresa.

** Vocal Asesor, Dirección General de Política Económica. Ministerio de Economía y Empresa.

Las opiniones expresadas en este artículo son responsabilidad exclusiva de sus autores y no representan necesariamente las de ninguna institución.

Versión de abril de 2019.

DOI: https://doi.org/10.32796/ice.2019.907.6809
} 


\section{Introducción}

La mejora de la regulación persigue garantizar que las medidas regulatorias cumplen con los objetivos previstos de las diferentes políticas públicas (medioambiental, social, laboral, etc.) al mínimo coste. Para ello, la mejora de la regulación se articula a partir de un conjunto de principios de actuación que deben tenerse en cuenta en cualquier intervención (necesidad, proporcionalidad, eficacia, eficiencia, seguridad jurídica y transparencia) ${ }^{1}$ y una variedad de instrumentos o herramientas que se implementan para garantizar el cumplimiento de dichos principios, como son la evaluación ex ante y ex post de las políticas públicas, los procesos de reducción de cargas administrativas o los mecanismos de prevención y detección de problemas regulatorios.

A lo largo de los últimos años, la adaptación y mejora de la regulación ha ido ganando espacio en la agenda de la Organización para la Cooperación y el Desarrollo Económico (OCDE), la Unión Europea (UE) y los Estados miembros (EE MM). En particular, en el ámbito europeo, y a pesar de que la mejora de la regulación no se circunscribe al ámbito económico, los esfuerzos han sido especialmente intensos en lo referido a la regulación del mercado interior. Así, de forma paralela a la constatación del potencial beneficio derivado de la mayor integración del mercado interior ${ }^{2}$ (Parlamento Europeo, 2017), se han ido desarrollando tanto políticas transversales (bajo etiquetas como la de Better o Smart Regulation) $)^{3}$, como aproximaciones

\footnotetext{
1 En España actualmente a nivel estatal los principios e instrumentos de la mejora de la regulación se encuentran recogidos principalmente en la Ley 39/2015, de 1 de octubre, del Procedimiento Administrativo Común de las Administraciones Públicas; la Ley 40/2015, de 1 de octubre, de Régimen Jurídico del Sector Público; y en el ámbito económico en la Ley 20/2013, de 9 de diciembre, de garantía de la unidad de mercado.

2 El Parlamento Europeo cifró el coste de la falta de integración del mercado de bienes en un $1,3 \%$ del PIB de la UE y en un $2,4 \%$ para el caso de los servicios.

3 Cabe señalar el intenso trabajo desarrollado tanto por la OCDE (2015a), como por la Comisión Europea (2017a). Resultan de interés los análisis realizados por la OCDE, recuperados de http://www.oecd.org/gov/ regulatory-policy/betterregulationineurope-progressandtrends.htm
}

sectoriales de buena regulación económica basadas en el desarrollo de los comentados principios e instrumentos. Como herramienta fundamental de estas políticas se han realizado procesos de análisis de los marcos regulatorios en vigor, con la aspiración de que los mismos permitan entender en qué medida las normas aprobadas responden a la razón que justificó la intervención y hasta qué punto los requisitos impuestos a los operadores se conforman o no en barreras para el libre acceso o ejercicio de las actividades económicas.

En el marco de dichos análisis, se han acumulado enfoques y experiencias que resultan valiosas de cara al perfeccionamiento de las metodologías usadas para conocer actividades cada vez más dinámicas y complejas. Con todo, a pesar de los avances, se aprecia la necesidad de continuar desarrollando las metodologías que se emplean y la complementariedad de fuentes con las que se trabaja, a fin de facilitar la elaboración de propuestas eficaces (Consejo de Competitividad, 2019) 4 .

El presente artículo se centrará especialmente en las metodologías aplicadas para la regulación económica, aunque el análisis comparta elementos comunes con las políticas de buena regulación general en sentido amplio. Así, tras una valoración sobre por qué es necesaria la mejora y sistematización de fuentes, se hará un repaso panorámico de las alternativas existentes, agrupando las mismas en función de su origen; esto es, disociando las que surgen de la perspectiva de la Administración, las que lo hacen a partir del usuario y las que recurren a datos microeconómicos. A partir de dicha clasificación se tratará de plantear las limitaciones y ventajas que se han observado en la práctica en procesos de adaptación normativa relevantes, como son los que han supuesto la Directiva 2006/123/CE del Parlamento Europeo y del Consejo, de 12 de diciembre de 2006, relativa a los servicios en

\footnotetext{
4 En el momento de la elaboración de este artículo el Consejo de Competitividad se dispone a incluir estas cuestiones en sus conclusiones (aún en fase de borrador). Ello constata la importancia que se va otorgando a la cuestión en los últimos años.
} 
el mercado interior -Directiva de Servicios- o la Ley 20/2013, de 9 de diciembre, de garantía de la unidad de mercado (LGUM).

2. Acotación del problema: la importancia de la mejora y sistematización de las metodologías de análisis

Si se hace una revisión de las temáticas tratadas en los foros de alto nivel en los últimos años, tanto en el ámbito de la UE como de los EE MM, se observa cómo el refuerzo del mercado interior ha sido protagonista habitual de las agendas de debate ${ }^{5}$. En este sentido, el avance en la integración de los mercados de bienes y servicios se ha articulado de forma transversal mediante políticas de buena regulación económica, línea en la que se ordenan la Directiva de Servicios o la LGUM.

En esencia, en el plano económico estas políticas, al igual que las de mejora de la regulación de carácter general, han consistido en la definición de unos principios básicos de regulación, entre los que destacan en este ámbito los de necesidad, proporcionalidad y no discriminación, y el desarrollo de herramientas específicas para garantizar su efectiva aplicación. Se trata, en suma, de desarrollar un marco teórico y unos instrumentos prácticos que permitan la adaptación de la cultura regulatoria, lo que requiere de un trabajo continuo de revisión, que garantice que las futuras normas se adaptan al nuevo marco y que las vigentes se evalúan y, en su caso, adecúan al mismo (Álvarez, M. y Domínguez, J., 2016).

La aplicación práctica de estos principios requiere, caso a caso, de un esfuerzo descriptivo de aquello que se pretende eliminar o adaptar. Esto es, se debe entender la lógica que hay detrás de cada requisito regulatorio y cómo afecta este realmente al operador,

\footnotetext{
5 La mejora del mercado interior es una de las diez prioridades políticas de la Comisión para 2015-2019, vinculando la aplicación efectiva de la buena regulación económica con la necesaria eliminación y prevención de barreras al acceso y ejercicio.
}

para analizar después en qué medida el mismo podría resultar innecesario o desproporcionado y por qué y en qué términos podría existir una alternativa menos gravosa. No obstante, esta labor descriptiva no resulta sencilla. Por una parte, se aprecia que las actividades económicas y los modelos de negocio presentan en muchas ocasiones un carácter dinámico y cambiante ${ }^{6}$, lo cual obliga a que la lectura deba anticipar dicha posible evolución, y a que la norma sea suficientemente flexible ${ }^{7}$. Por otra parte, es común que la competencia de regulación se reparta entre múltiples niveles, lo que lleva a que la lectura de necesidad y proporcionalidad pueda no resultar unívoca o consistente entre las distintas autoridades competentes. Esto provoca que los Tribunales se conviertan en última instancia en intérpretes y garantes últimos de los principios de buena regulación económica ${ }^{8}$.

Por todas estas razones, resulta imperativo consolidar metodologías precisas que permitan obtener información suficiente que quede recogida en los análisis de impacto, tanto ex ante como ex post. Se trata con todo ello de que todas las partes -operadores, Administraciones y Tribunales - conozcan de forma precisa la realidad sobre la que se interviene, el modo en que se articula la intervención y los efectos que genera, de manera que se pueda valorar de una forma adecuada la idoneidad de la medida.

Hoy se reproducen, impulsadas por las distintas Administraciones, aproximaciones metodológicas que se caracterizan en particular por la fuente de información utilizada. Así, se podría hablar de: aproximaciones top-down o clásicas, para aquellos casos en los que la fuente de información es la propia Administración interviniente; la aproximación bottom-up, en la

\footnotetext{
6 Esta dificultad, que resulta especialmente evidente en el caso de modelos de negocio disruptivos (OCDE, 2015a), puede bien hacerse extensible al conjunto de actividades. Véase Comisión Europea (2018a).

7 La cuestión de la regulación adaptable a futuro «Normas preparadas para el futuro» se ha convertido en una aspiración que se reitera en el ámbito comunitario (Comité Económico y Social, 2016).

8 Se puede consultar información sobre las sentencias en materia de LGUM en http://www.mineco.gob.es/portal/site/mineco/gum
} 
que la información proviene del operador afectado por la regulación; o la aproximación sobre datos objetivos -información micro-, con la que tratamos de describir la actividad y comportamiento de los agentes intervinientes. Todas ellas cuentan con potenciales ventajas e inconvenientes, $y$ han sido objeto de procesos de revisión, en busca de un mejor reflejo de la actividad que tratan de describir.

\section{Metodologías de análisis a partir} de la perspectiva de la Administración: la aproximación top-down

La aproximación de detección de problemas regulatorios basado en un análisis top-down (proceso de toma de decisiones de arriba-abajo) engloba aquellas técnicas basadas en el modelo clásico o tradicional de funcionamiento de la Administración.

De este modo, es la propia Administración la que a su juicio y criterio selecciona los problemas (las posibles barreras u obstáculos a la actividad económica) sobre los que focalizar su atención y priorizar su actuación. Asimismo, es la Administración la que desarrolla el análisis de la actividad económica a regular desde su propio prisma, y decide la forma óptima de intervención. El motor del cambio regulatorio y del impulso de la mejora de la regulación es, en suma, la propia Administración.

Este tipo de aproximaciones se han enriquecido, además, con el desarrollo de análisis comparados en los que las distintas Administraciones contrastan la solución que han dado para un mismo problema. En esta línea, destaca el análisis en diversos foros internacionales, como es el caso del Banco Mundial, con el desarrollo de los indicadores incluidos en el Doing Business ${ }^{9}$.

A fin de enumerar la variedad de técnicas de análisis existentes, la praxis de la UE resulta especialmente

\footnotetext{
9 Se puede consultar informes y metodología en http://www. doingbusiness.org/en/doingbusiness
}

útil. Así, al margen de los foros en los que participan los Estados en su función de colegislador (a través del Consejo o la comitología), podemos encontrar las siguientes estructuras de análisis:

- Formatos de revisión y análisis ad-hoc, tipo cluster, donde se realizan ejercicios de revisión o evaluación mutua entre los diferentes EE MM de la transposición de normativa comunitaria o de intercambio de buenas prácticas para la regulación de alguna actividad. Ejemplo de ello son los ejercicios habitualmente realizados en el marco de la Directiva de Servicios o en el de profesiones reguladas (Álvarez, M., 2013).

- Canales telemáticos de monitorización y revisión mutua, como son los articulados a través de los mecanismos de notificación. Se trata de procedimientos reglados a nivel comunitario a fin de que los EE MM notifiquen a través de plataformas telemáticas los requisitos aprobados para ciertas materias (como son las reglamentaciones técnicas y requisitos de servicios de la sociedad de la información). Con ello, se promueve la transparencia y supervisión mutua ex ante entre Administraciones, a fin de evitar la interposición de barreras regulatorias (Díaz Mesanza y Medina Asensio, 2017).

- Los grupos de alto nivel, grupos de expertos o foros de debate sectoriales. Estos foros, que pueden o no tener carácter permanente, tienen como objetivo la generación de análisis y debate en torno a determinadas temáticas. A modo de ejemplo podrían citarse, entre muchos otros, el Grupo de Alto Nivel de Competitividad y Crecimiento y el Grupo de Expertos de Directiva de Servicios, a nivel comunitario, o los Grupos de Trabajo de Conferencias Sectoriales, a nivel nacional.

En cualquier caso, a pesar de sus diferencias y de su variable configuración, se aprecian elementos comunes. Así, en cuanto al enfoque, todas estas formas de cooperación se centran en prioridades específicas dentro de la agenda política y todas se orientan esencialmente a proporcionar conocimiento especializado a través del análisis de los problemas regulatorios 
detectados y de las posibles soluciones para mejorar la regulación.

Además, respecto a su conformación, todos estos formatos quedan participados fundamentalmente por las Administraciones Públicas ${ }^{10}$, con independencia de que estos instrumentos hayan ido evolucionando para adoptar fórmulas más o menos abiertas que permitan la toma en consideración de otros agentes interesados. De este modo, entre las modalidades usadas recientemente, encontramos formatos tipo stakeholder exercise, como el llevado a cabo a través del Single Market Forum (Comisión Europea, 2017b); comités consultivos o de diálogo con la sociedad civil (habituales, por ejemplo, en el ámbito de la agricultura y el mundo rural); o el encargo de estudios de consultoría, p. ej., entre muchos otros, el relativo al análisis de barreras normativas al establecimiento en el sector del comercio (Comisión Europea, 2016). Todo ello permite una evolución de estos instrumentos, de forma que el enfoque top-down se acerca y se complementa con elementos de aproximaciones tipo bottom-up, que serán explicados en el siguiente apartado. No obstante, ello no desvirtúa el carácter esencial de este enfoque primario, en la medida en que sigue siendo la propia Administración la que decide a quién dirigirse y en qué términos se realiza el análisis.

En España, esta dinámica de análisis top-down se conforma en praxis habitual, a través de los órganos de cooperación interadministrativa que se encuentran regulados por la Ley 40/2015, de 1 de octubre, de Régimen Jurídico del Sector Público (que, de hecho, prevé esa composición específicamente administrativa a la que se hacía referencia). Así, se posibilita la existencia de diferentes formatos, como son las conferencias sectoriales (de nivel político, pero con la capacidad de establecer grupos de trabajo técnicos) u otros órganos de cooperación. En la actualidad existen unas 40 conferencias sectoriales, aunque su actividad (formato,

\footnotetext{
10 Sirva de ejemplo a nivel comunitario el Grupo de Expertos de la Directiva de Servicios (Comisión Europea, 2019).
}

análisis realizados y tipo de acuerdos adoptados) es muy variable (MPAT, 2016).

Al igual que en el ámbito europeo, estos mecanismos se han complementado con otros instrumentos. Por ejemplo, a imagen y semejanza de los mecanismos de notificación comunitarios, el artículo 14 de la LGUM ${ }^{11}$ prevé un sistema de cooperación e intercambio de proyectos normativos a través de un sistema electrónico de información, previsto en la propia ley. Su utilidad, sin embargo, está siendo aún limitada, lo que puede ser debido a que la compartición de proyectos parece descansar en la voluntariedad de las Administraciones y a que el sistema no es actualmente accesible a los entes locales. Así, a fecha de 11 de marzo de 2019 y desde su puesta en marcha en 2015 , se han colgado en dicha plataforma un total de 183 proyectos normativos (33 correspondientes a la Administración General del Estado y 150 a comunidades autónomas). Si bien los mismos representan una pluralidad de ámbitos, parecen predominar algunos sectores concretos, como son la industria a nivel del Estado, y el turismo, la sanidad, el medioambiente, el juego, los asuntos sociales y la agricultura y pesca a nivel autonómico.

En cualquier caso, la experiencia acumulada por el reiterado uso de estas fórmulas de revisión, tanto en el marco de la LGUM como de la Directiva de Servicios, pone sobre la mesa algunas ventajas pero también ciertos déficits que cabe tener en cuenta.

Las ventajas resultan del papel privilegiado de la Administración, en tanto que regulador. Así:

- Permite una comprensión precisa de la racionalidad a la que atiende la norma; esto es, permite entender en qué medida la norma final, y la implementación que de la misma se hizo, respondió al objetivo original

\footnotetext{
11 La LGUM contiene diferentes disposiciones para fomentar la cooperación interadministrativa; se dota de nuevas funciones a las conferencias sectoriales (artículo 12); se crean nuevos mecanismos de cooperación para la integración de la información de registros, la compartición de proyectos normativos y para facilitar las comunicaciones entre autoridades (artículo 23); y se crea el Consejo para la Garantía de la Unidad de Mercado.
} 
del regulador. Ello es así por ser resultado de una valoración en primera persona (por el regulador).

- Permite la comparación entre esas mismas lógicas regulatorias, mediante debates estructurados: análisis técnicos participados por funcionarios con conocimiento especializado y a partir de ejemplos prácticos, y el intercambio de buenas prácticas entre las diferentes Administraciones Públicas. Eso posibilita una más fácil identificación de los posibles riesgos y de las opciones regulatorias a tomar.

- Favorece el impacto práctico, dado que las conclusiones que se adoptan son susceptibles de encontrar acomodo inmediato en la agenda del regulador. Ello resulta del hecho de estar conformadas por Administraciones cuyo actuar (ya sea en forma de análisis y propuestas) responde en última instancia a un desideratum político ${ }^{12}$.

Con todo, el uso de estas fórmulas cuenta con algunos inconvenientes:

- El hecho de que respondan a desideratum político condiciona los temas que se abarcan. Bien sea por miopía en la detección de barreras (desconocimiento del impacto práctico), por condicionantes en el establecimiento de prioridades o por falta de voluntad política para abordar cuestiones controvertidas, existen temas que quedan al margen del análisis a pesar de tener incidencia práctica notable. Buen ejemplo de ello han sido las economías colaborativas donde, ya sea por complejidad técnica o por implicaciones políticas, se ha producido una intervención tardía por parte de las Administraciones (Comisión Europea, 2018c).

- Por su propia composición (miembros de la Administración Pública) estos mecanismos tienden a centrarse en problemas u obstáculos de carácter estrictamente regulatorio, frente a los de carácter no regulatorio, más difíciles de detectar. Asimismo, el análisis puede tener tendencia a centrarse en debates

\footnotetext{
12 Ejemplo de ello son el Paquete de Servicios en el ámbito comunitario o las reformas normativas resultantes del Programa de Adaptación Normativa que se desarrolló con la LGUM, en el ámbito nacional.
}

«teóricos» cuya incidencia real sea mínima o desconocida, por falta de datos que fundamenten el análisis. En definitiva, su análisis puede ser parcial y alejado de la realidad.

- Para el caso de actividades muy transversales, en las que quedan afectados distintos niveles administrativos y materias, la formalización de los debates, realización del análisis y adopción de conclusiones resulta compleja (INAP, 2015). Así, por ejemplo, analizando la actividad de las conferencias sectoriales en los últimos años puede apreciarse que el número de acuerdos finales adoptados con incidencia clara en la regulación de las actividades económicas es muy escaso (básicamente en sectores como juego, consumo, turismo o industria) ${ }^{13}$. Del mismo modo, las diferencias entre los contextos regulatorios de las Administraciones participantes limitan la exportabilidad de las buenas prácticas analizadas.

La existencia de estas limitaciones suscita la necesidad de complementar este análisis con fórmulas que permitan mejorar la comprensión de la regulación y su impacto.

\section{Metodologías de análisis a partir de la participación de los operadores: la aproximación bottom-up}

La aproximación de detección de barreras a través de la participación de operadores y ciudadanos consiste en una inversión de la lógica de las fórmulas de análisis clásicas descritas anteriormente y son conocidas como aproximaciones bottom-up (por articularse de abajo-arriba). Estas, en esencia, consisten en valoraciones de la adecuación del marco en función de la perspectiva del usuario.

Siguiendo este enfoque, son los destinatarios de la norma en sentido amplio los encargados de señalar los problemas en los que la Administración debe focalizar

\footnotetext{
${ }_{13}$ Véanse al respecto los informes de actividad de las conferencias sectoriales, elaborados anualmente por el ministerio competente.
} 
su atención. La capacidad de impulso de la mejora de la regulación descansa, por tanto, en la movilización de los agentes, que se convierten en protagonistas y motor del cambio.

Estas aproximaciones parten de los instrumentos desarrollados con la evolución de la orientación de la Administración al ciudadano y con el arraigo del papel proactivo del mismo. Las herramientas clásicas, que podrían considerarse «de primera generación», serían instrumentos como la revisión en vía administrativa de los actos de la Administración, las consultas públicas preceptivas en el proceso de elaboración normativa ${ }^{14}$, o el amplio derecho de petición. Sin embargo, en los últimos años se han ido desarrollando nuevas capacidades y derechos de comunicación y transparencia activa. Asimismo, se ha extendido el conocimiento de los usuarios de los procedimientos especiales de queja en materia económica y de sus derechos y los estándares de calidad de servicios que debe prestar la Administración ${ }^{15}$. Todo ello deriva en un conjunto amplio de fuentes de información a la hora de detectar los problemas regulatorios y no regulatorios que, en la práctica, afectan a los operadores.

El enfoque bottom-up se caracteriza así por cierta naturaleza híbrida en la que, mientras son los operadores los que determinan cuál es el problema a resolver, es la Administración mediante diferentes vías de cooperación la que debe solucionar el mismo. Ejemplos a nivel nacional de mecanismos bottom-up más desarrollados que podrían, por tanto, ser considerados de "segunda generación» serían los mecanismos de protección de operadores de los artículos 26, 27 y

\footnotetext{
${ }^{14}$ Estos procesos de consulta pública —no restringidos únicamente al proceso normativo - se han ido reforzando progresivamente. Así, se ha ido pasando de procedimientos de audiencia limitados a procesos más amplios de información pública e incluso en distintas fases de la tramitación, por ejemplo mediante la «consulta pública previa».

15 Estas medidas de potenciación del rol de operador conforman uno de los valores añadidos de la LGUM y el centro sobre el que giran muchas de las acciones de la estrategia de mercado interior, desarrolladas tanto a través del Service Package como del Enforcement Package (Comisión Europea, 2018a).
}

28 de la LGUM $^{16}$, las reclamaciones ante el Consejo de Transparencia y Buen Gobierno o los sistemas de quejas y sugerencias creados por el Real Decreto $951 / 2005$, de 29 de julio, por el que se establece el marco general para la mejora de la calidad en la Administración General del Estado.

Instrumentos que también han contado con cierto alcance en la UE serían el mecanismo de solución de problemas «SOLVIT» ${ }^{17}$ o los procedimientos de infracción en fase previa «Proyecto Piloto» planteados por denuncia de parte.

Una aproximación panorámica del uso de estas fuentes las aporta el Gráfico 1, que refleja las quejas, reclamaciones o denuncias recibidas por los distintos mecanismos -entendidos en sentido amplio- en el año 2017, según sus memorias o informes anuales tanto a través de canales europeos, en forma de reclamaciones frente al Defensor del Pueblo Europeo y reclamaciones de operadores «vía SOLVIT o EU Pilot», como de instrumentos análogos en España (particularmente las reclamaciones al Consejo de Transparencia en España y las reclamaciones en virtud de los comentados artículos 26,27 y 28 de la LGUM). Aunque, obviamente, su uso dependa en gran medida del ámbito en el que opera cada mecanismo, las cifras reflejan una reducida utilización de todos ellos —ninguno llega a más de 2.500 quejas anuales_, lo que contrasta con el elevado recurso al Defensor del Pueblo de España (24.976 quejas en 2017 frente a por ejemplo las 2.181 a nivel europeo) o a los más accesibles y conocidos sistemas de quejas y sugerencias en España (44.009 recibidas en 2017).

\footnotetext{
16 La LGUM establece una vía formal de carácter alternativo al sistema administrativo de recursos (procedimiento del artículo 26) y un mecanismo adicional que opera como procedimiento de arbitraje (artículo 28 de la LGUM). Además, el artículo 27 de la LGUM legitima a la Comisión Nacional de los Mercados y la Competencia (CNMC) para impugnar actos y disposiciones ante la Audiencia Nacional (Álvarez, M. y Balsa, C., 2017).

17 SOLVIT es un sistema de resolución de problemas formado por una red de centros nacionales de los Estados miembros cuyo objetivo es atender de forma rápida los problemas transfronterizos del mercado único planteados por ciudadanos y empresas.
} 


\section{GRÁFICO 1}

USO DE MECANISMOS BOTTOM-UP, 2017

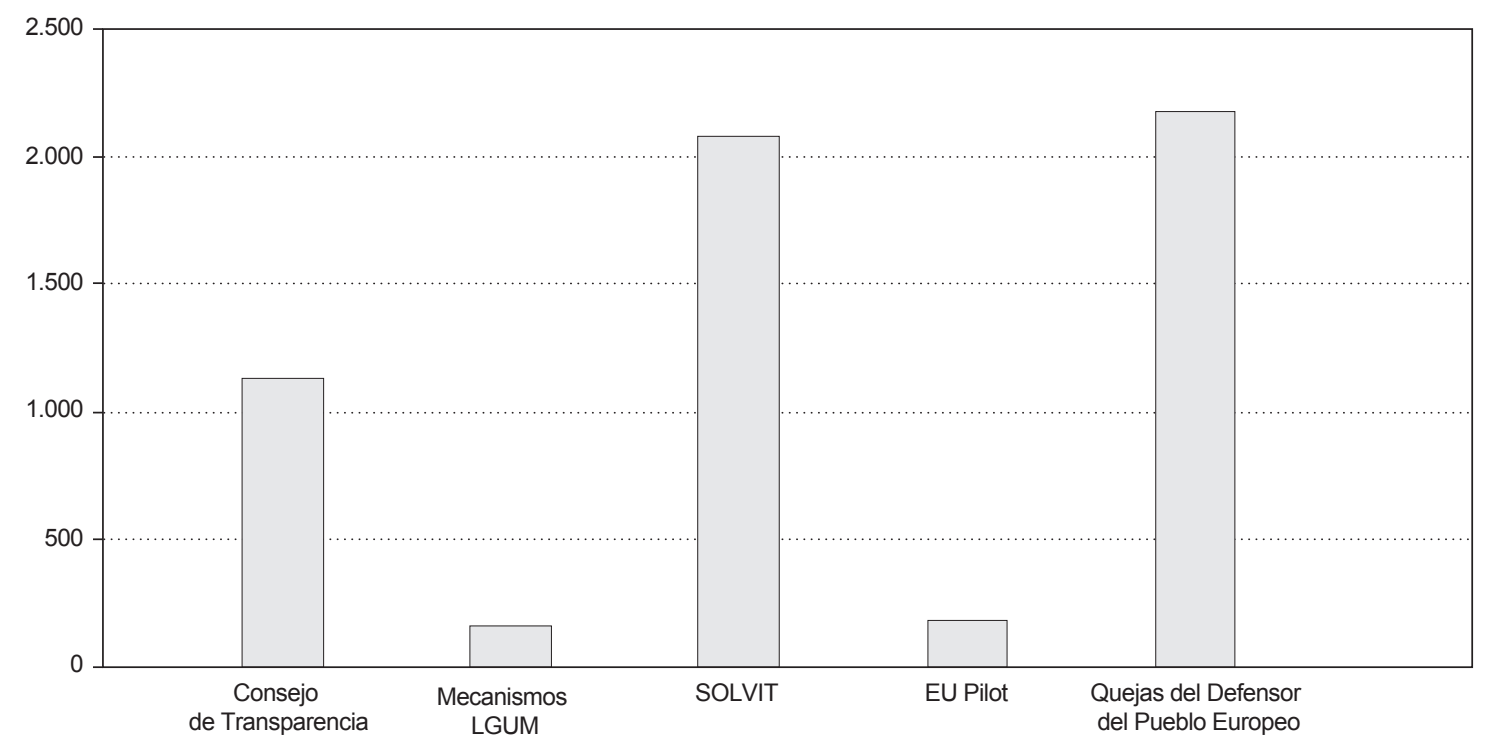

FUENTE: Memorias, informes o datos oficiales de los diferentes mecanismos.

Con todo, aunque en términos absolutos el uso de dichos mecanismos — por ejemplo los de la LGUM — pueda considerarse limitado, el análisis agregado del uso de los instrumentos 26 y 28 de la LGUM desde su creación, según sector, nos ofrece una buena aproximación de aquellos ámbitos de actividad en los que los operadores consideran que persisten problemas o barreras regulatorias. Así, por ejemplo, en los cinco años de uso de los mecanismos de la LGUM se han presentado casos en múltiples sectores aunque la mayoría de las reclamaciones pertenecen al ámbito de los servicios profesionales, el transporte, las telecomunicaciones, el comercio y ayudas a la formación del empleo (Gráfico 2).

Del mismo modo, en el ámbito europeo, por ejemplo, las reclamaciones recibidas por SOLVIT reflejan que los ámbitos donde persisten problemas en las relaciones transfronterizas entre Estados miembros están relacionados con los trámites de la Seguridad Social, el reconocimiento de cualificaciones profesionales, la libre prestación de servicios y los permisos de residencia.

En cualquier caso, estas fuentes de información han sido objeto de constante evolución. Así, se han venido complementando con técnicas que buscan suministrar información de detalle para áreas concretas de actividad sobre las que se quiere acumular conocimiento. En este sentido, se observa un creciente uso de técnicas de aprendizaje por suplantación u observación del usuario de los servicios públicos, a imitación de técnicas de investigación de mercados y marketing, como por ejemplo el mystery shopper o shadowing, mediante las cuales trata de aproximarse al impacto práctico de las normas, evaluándose posibles trabas sobre el operador o nivel de impacto en términos de protección del consumidor. Dado el nivel de detalle que se puede extraer del análisis, se ha producido una progresiva extensión en el empleo de estas técnicas. Así, entre otros ejemplos, se puede mencionar la 


\section{GRÁFICO 2}

\section{CLASIFICACIÓN CASOS 26/28 LGUM POR SECTOR}

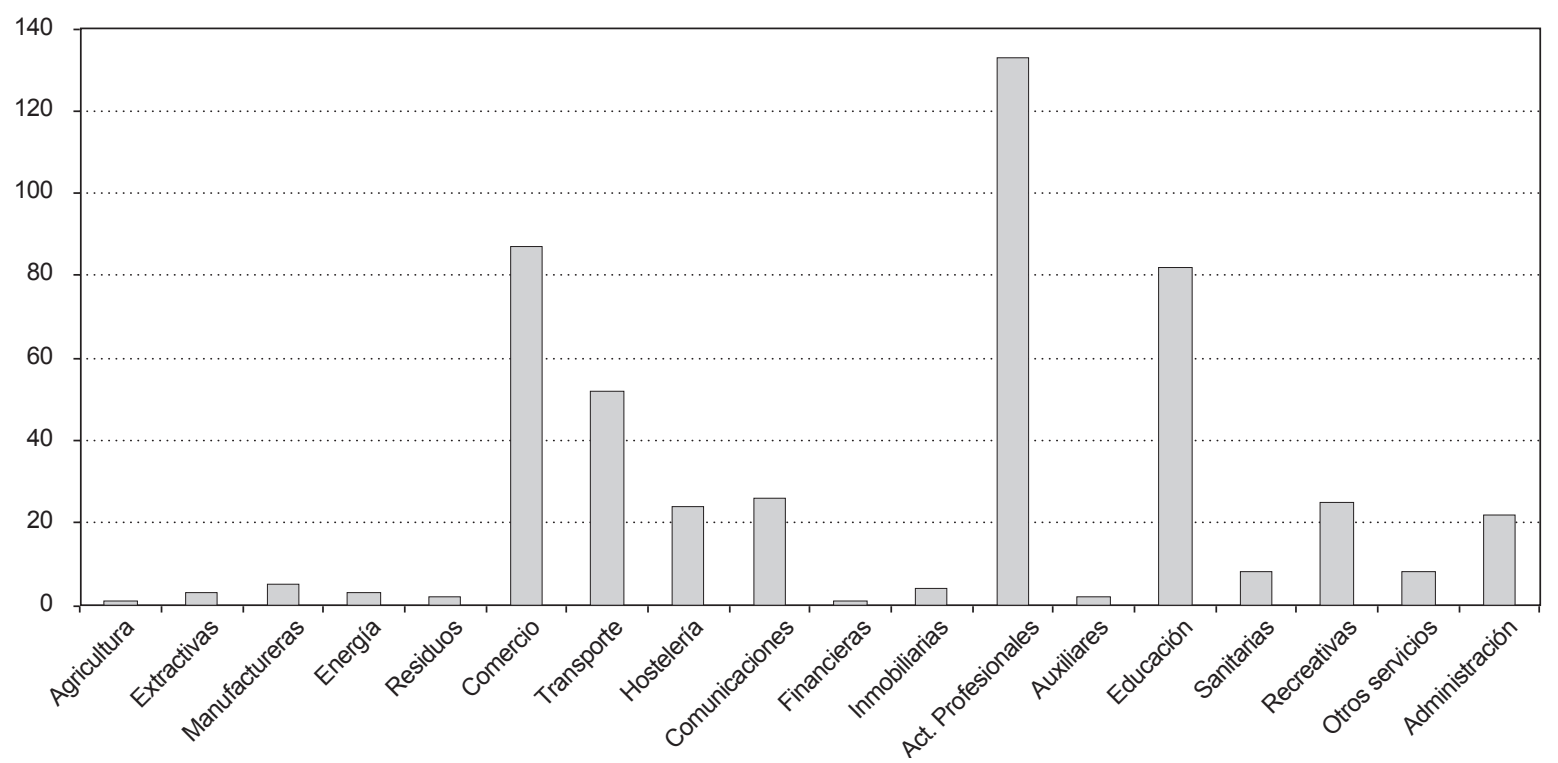

FUENTE: Secretaría del Consejo para la Unidad de Mercado.

aplicación de esta técnica en Reino Unido para detectar los problemas en contratación pública (Gobierno de Reino Unido, 2017) o, con carácter nacional, el uso de estas técnicas a efectos de control, como ejemplifican los casos de la Comisión Nacional del Mercado de Valores para la venta de productos financieros en oficinas bancarias (CNMV, 2017) o por la Comisión Nacional de los Mercados y la Competencia (CNMC) para las empresas suministradoras de gas (CNMC, 2018).

Las ventajas asociadas a estas aproximaciones bottom-up son varias:

- Permiten una definición de la agenda centrada en problemas prácticos y efectivos. Restan, por tanto, abstracción al debate y obliga a la inclusión en la agenda de las temáticas complejas por resultar controvertidas políticamente o por ser difíciles de abordar técnicamente.

- Permiten la comprensión de la carga regulatoria que se ejerce de conjunto del operador, sin importar el reparto por materia o territorio entre distintas Administraciones. Ello resulta fundamental a la hora de instrumentar propuestas.

Con todo, se aprecian algunas dificultades o carencias en la aplicación de estos modelos:

- En ocasiones, las aportaciones quedan sesgadas por la defensa de intereses de parte. Así, el análisis realizado puede estar distorsionado por el empuje de determinados grupos de interés. Los canales de comunicación de la Administración de la ciudadanía son usados de forma profusa por los grupos lobbies con mayores medios y mejor organizados o por interesados específicos con motivaciones muy concretas. Esto puede generar que la identificación de problemas y el debate se oriente hacia cuestiones cuyo interés general es relativo. Es decir, estos mecanismos permiten focalizar el análisis hacia problemas reales pero cuya incidencia puede ser parcial. Así, en el caso de los mecanismos de reclamación de la LGUM, el ámbito subsectorial con 
mayor número de reclamaciones (un $16 \%$ de las presentadas) es el de los centros de formación de empleo; sin embargo, todas estas denuncias (más de 50) fueron presentadas por un único operador.

- Estos mecanismos están creados para que la sociedad civil sea "oída», pero que sea "escuchada» depende de la voluntad administrativa y política. Así, por ejemplo, los mecanismos de la LGUM han puesto de manifiesto que en España existe un problema de determinación de competencias entre determinadas profesiones -el ámbito de servicios profesionales es el sector global con mayor número de reclamaciones (Gráfico 2)-, concentrándose estas en el ámbito de profesiones técnicas, básicamente entre arquitectos e ingenieros. Sin embargo, no se ha producido ninguna iniciativa en los últimos años orientada a la solución de este problema sin perjuicio de las propuestas que provienen de iniciativas comunitarias.

- Cuando se articulan en formato foro de debate estructurado $^{18}$, resultan costosos de aplicar. Estructurar debates fluidos con operadores resulta difícil, en tanto que se requiere de la detección previa de operadores en el área de la actividad a analizar que conformen una muestra adecuada y que tengan voluntad de participar.

De este modo, cabe plantearse el recurso adicional a fuentes de información no subjetivas. Llegamos así al análisis del dato: las fuentes microeconómicas.

\section{Análisis microeconómico}

El enfoque micro del dato se diferencia de las anteriores aproximaciones en que trata de objetivar el análisis a partir de la descripción del comportamiento de

\footnotetext{
18 Se trata de debates en los que se estructura la discusión sobre temáticas concretas y cerradas - para las que se han realizado previamente encuestas o análisis-, como sucede, por ejemplo, en los desarrollados en el marco del Single Market Forum. Estos resultan complejos y costosos por requerir la participación de agentes que sepan reconocer y transmitir con cierta precisión los problemas regulatorios que experimenta la empresa a la hora de desarrollar su actividad. Además estos deben representar la diversidad de operadores presentes en el mercado para ver cómo repercuten estos según cada tipología.
}

los operadores en sus interacciones con las Administraciones y en el propio mercado.

En esencia, lo que permite esta fuente de información es entender cuestiones básicas sobre la actividad a regular, mediante la caracterización de qué tipo de operadores acceden al mercado (procedencia, tamaño, volumen de negocio, etc.), cómo lo hacen (de forma temporal o permanente) y qué tipo de interacciones han de efectuar con la Administración y a qué coste (asesoramientos, trámites y controles por parte de las Administraciones intervinientes).

Todas estas variables son relevantes de cara a efectuar la valoración del cumplimiento de los principios de buena regulación económica del marco normativo. Así, por ejemplo, no resulta análoga una valoración del impacto económico de un trámite para un mercado con altos costes de entrada en el que solo operan grandes empresas que para un mercado en el que todo lo que operan son microempresas.

El desarrollo de estas metodologías basadas en el dato se ve limitado por las dificultades para obtener la información, motivo por el cual tradicionalmente las memorias de análisis de impacto normativo (MAIN) han encontrado en la justificación de proporcionalidad una de sus principales carencias (Comisión Europea, 2016).

En esta línea, el enriquecimiento de estas fuentes se ha conformado como una prioridad para las Administraciones. Así, basta señalar los esfuerzos de la Comisión Europea, que marcó como prioridad el refuerzo del análisis de impacto, dentro de la Agenda de Mejora de la Regulación (Comisión Europea, 2015) o la propuesta de la nueva herramienta de información del mercado interior - Single Market Information Tool(Comisión Europea, 2017b), mediante la cual la Comisión aspira a obtener información objetiva procedente del propio usuario: la empresa, con el fin de detectar malos funcionamientos del mercado. Se ahonda así en una tendencia que trata de garantizar el soporte en datos objetivos de los juicios técnicos.

Es precisamente en este punto donde debe destacarse la oportunidad que genera la digitalización. 
A medida que los negocios y las transacciones que generan los mismos pasan por la Red, resulta cada vez más factible extraer datos agregados que permitan caracterizar a los agentes y sus interacciones. Esto es, mediante explotación de grandes volúmenes de datos se puede aspirar a entender mejor realidades cada vez más complejas.

Aunque tímidamente, ya encontramos ejemplos de Administraciones utilizando estas fuentes. Así, lo demuestra el ejemplo de los nuevos modelos de negocio, y en particular para el caso del análisis de la regulación de la vivienda vacacional, para el que distintas Administraciones se han venido apoyando en el uso de estas nuevas fuentes de datos que permiten conocer la realidad sobre la que se pretende regular. En este sentido, se han apoyado en servicios web de explotación de big data para obtener información sobre cuántas viviendas se ofrecen en una plataforma, por cuántos días, con qué ingreso medio y si es la única vivienda ofertada por el operador. Todo ello se trata de información que resulta útil de cara a entender o acercarse al incentivo a la inversión especulativa en el sector, al volumen de vivienda que puede sustraer del mercado residencial o a la concentración potencial del fenómeno ${ }^{19}$.

Con todo, la digitalización no llega a resolver todos los problemas sobre la disposición de fuentes de información. Muchos modelos de negocio no permiten un trazado digital por quedar sus transacciones al margen de las plataformas. Para estos casos, la única fuente de extracción de datos es el propio operador. Así, las patronales juegan un papel esencial y su fluida interrelación con las Administraciones resulta fundamental, línea que se ha ido explotando en España en la elaboración de interesantes análisis como el desarrollado por la CEOE y la Secretaría de Estado de Comercio, en su informe anual de línea abierta (CEOE, 2017).

\footnotetext{
19 Se trata así de datos objetivos sobre los que las instituciones ya soportan sus argumentos, como así se ejemplifica en el Estudio de la Comisión sobre vivienda de uso turístico (Comisión Europea, 2018d) o el del Ayuntamiento de Madrid para esta materia (Ayuntamiento de Madrid, 2017).
}

En suma, la experiencia reciente refleja para estas fuentes algunas ventajas que la hacen susceptible de complementar los enfoques anteriormente citados:

- Los datos objetivos permiten la observación de comportamientos que escapan al conocimiento o apreciación subjetiva o práctica de los agentes (Administraciones u operadores), siendo una herramienta de análisis valiosa por sí misma.

- Adicionalmente, hace viable un análisis de consistencia de las conclusiones que se extraigan mediante el sistema bottom-up o top-down.

Como carencia, no obstante, se debe reseñar la gran dificultad para encontrar fuentes fiables y para integrar estas fuentes sin afectar a la serie histórica. Asimismo, debe tenerse en cuenta que para el tratamiento del dato y la elaboración de proyecciones es probablemente necesario el uso de técnicas o disciplinas ( $p$. ej., de tipo econométrico o basadas en la economía conductual) que pueden no estar al alcance de todas las Administraciones.

Del mismo modo, cabe recordar, en todo caso, que el dato, obtenido a través de técnicas adecuadas y solventando posibles problemas metodológicos, es intrínsecamente objetivo, pero el uso y la interpretación de este puede ser parcial y subjetivo.

\section{La importancia de la complementariedad de fuentes}

De una apreciación global de las ventajas y los inconvenientes de las distintas aproximaciones analizadas se infiere la necesidad de hacer un uso conjunto de estas a fin de favorecer un conocimiento completo de la actividad a regular. Así, es precisamente esa explotación conjunta la que permite, no solo tener una visión completa, sino además validar la consistencia del análisis. Por poner un ejemplo, resultaría lógico que cualquier aproximación top-down que aportase una prelación de países o regiones por nivel de restricciones se cruzase con datos de mercado, tales como los índices de penetración, a fin de determinar en qué medida las barreras impiden el acceso (p. ej., en caso de regiones muy restrictivas pero 


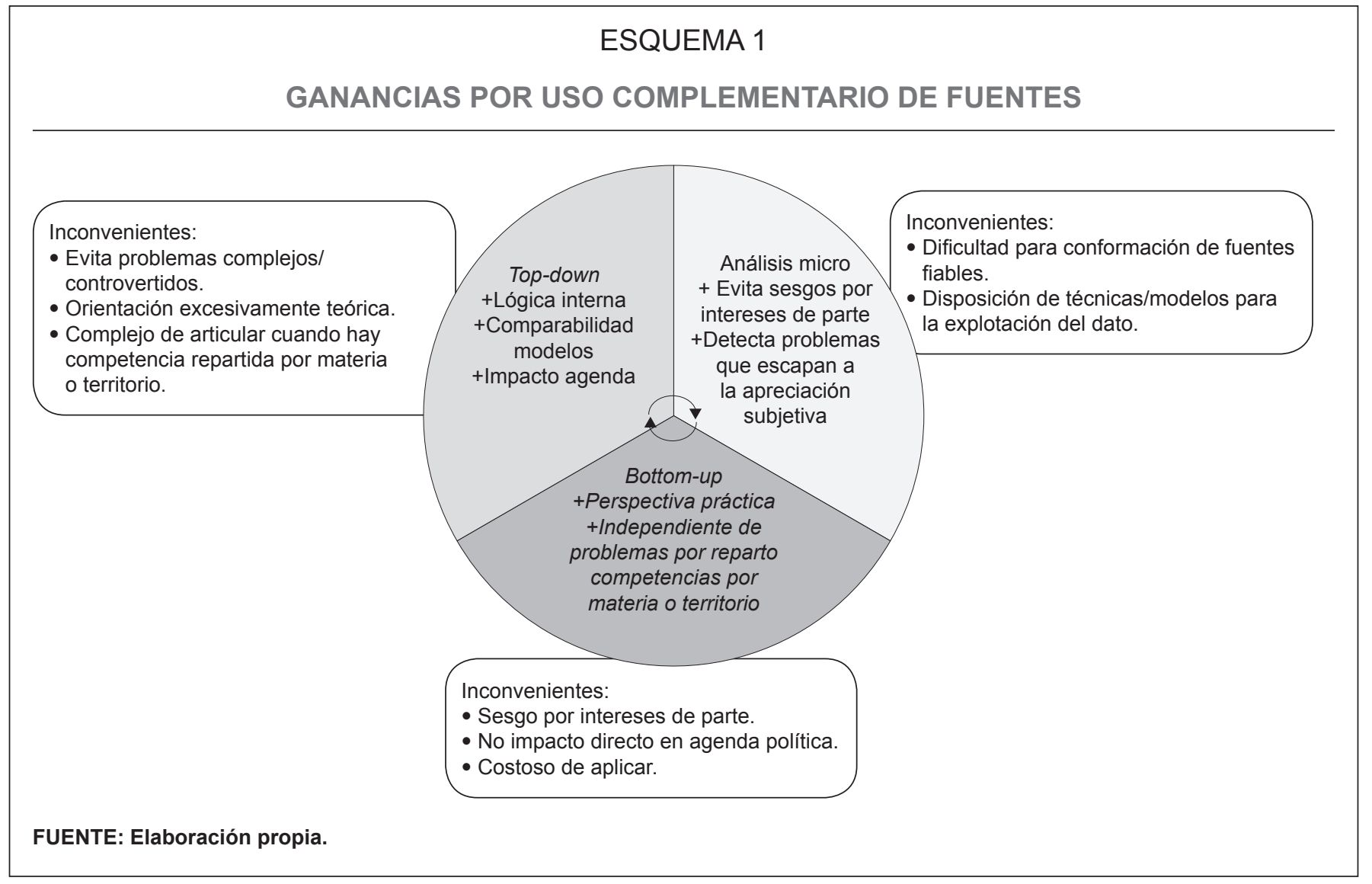

con altas tasas de penetración) o hasta qué punto el análisis es completo (p. ej., en supuestos con escasas restricciones y nula penetración de mercado). Si bien es un planteamiento sobre el que se trabaja, son comunes los ejemplos en los que no se desarrolla esta complementariedad (Comisión Europea, 2016).

Con todo, se ha ido avanzando en la potenciación de complementariedades, particularmente notables en el caso de las aproximaciones top-down y bottom-up, tanto a nivel comunitario, como ejemplifican los múltiples foros de análisis citados en este artículo, como a nivel nacional. De esta forma, en España, gran parte del valor añadido de la LGUM ha residido en ese refuerzo, en el que el trabajo de análisis interadministrativo que se articula en las conferencias sectoriales o en el resto de mecanismos de cooperación interadministrativa previstos en la norma podría ser reforzado con el análisis de la información que aportan los operadores con sus reclamaciones, lo que permitiría orientar la agenda a problemas prácticos.

En esta línea, parece que una de las principales áreas de mejora sigue siendo la complementariedad de estas fuentes con la óptica microeconómica, mediante la identificación y desarrollo de fuentes fiables y el desarrollo de modelos de tratamiento de las mismas, de forma que aporten soporte al desarrollo de los análisis de proporcionalidad y permitan validar la consistencia de las conclusiones de los análisis regulatorios que se hayan desarrollado (Esquema 1).

\section{Conclusión}

La buena regulación aplicada al ámbito económico supone que toda actuación debe quedar informada por 
una serie de principios básicos, entre los que destacan el de necesidad y proporcionalidad y el de no discriminación. La aplicación de estos principios requiere de un entendimiento completo del comportamiento de los agentes, de la singularidad de la actividad a regular y de las alternativas con que se cuenta para hacerlo.

La correcta comprensión de estos elementos ha generado la necesidad de desarrollar metodologías que se particularizan por el prisma que adoptan (el de la Administración, en análisis top-down, el usuario, en aproximaciones bottom-up, o a través del análisis del dato objetivo). No obstante, y a pesar del uso extendido de todas estas aproximaciones, la experiencia reciente tanto en el análisis de las barreras de mercado interior a nivel comunitario como en el de las trabas a la unidad de mercado a nivel nacional demuestra que hay espacio para la mejora. Este avance debe llegar no solo mediante el refinamiento de las técnicas utilizadas, camino en el que se orientan enfoques novedosos como el mystery shopper o la explotación de big data en los análisis micro, sino también mediante un refuerzo del uso complementario de las distintas aproximaciones disponibles. En este sentido, parece que aún queda recorrido para confrontar las aproximaciones top-down y bottom-up con la del dato microeconómico, algo fundamental a la hora de transmitir una imagen fiel de la realidad que se regula y para garantizar la consistencia de las conclusiones obtenidas.

A nivel nacional, la LGUM ha supuesto un avance importante en el uso de las diferentes metodologías de forma complementaria. Así, importando y desarrollando experiencias de la UE, la LGUM ha conseguido conjugar los análisis clásicos desde el prisma administrativo con la introducción de instrumentos como los mecanismos de protección, que permiten al operador orientar el análisis hacia las barreras que en la práctica le suponen problemas para el acceso o ejercicio de su actividad. Con todo, este modelo de mejora de la regulación económica aún adolece de una falta de fuentes fiables de datos y disposición técnica suficiente por parte de todas las Administraciones para su correcta explotación, lo cual se conforma como uno de los desafíos de cara a futuro.

\section{Referencias bibliográficas}

Álvarez, M. (2013). La Directiva de Servicios: 4 años después. Boletín Económico de Información Comercial Española, (3.046), diciembre, 31-40, Madrid.

Álvarez, M. y Balsa, C. (2017). Mecanismos de la Ley de garantía de la unidad de mercado. La buena regulación al alcance de los operadores. Estudios sobre la Ley de garantía de la unidad de mercado, 491-515, Madrid.

Álvarez, M. y Domínguez, J. (2016). La mejora de la regulación de los servicios en la Unión Europea y en España. Economistas, (150), Madrid. Ed. Colegio de Economistas de Madrid, 53-60.

Ayuntamiento de Madrid (2017). Análisis del impacto de las viviendas de uso turístico en el distrito Centro, Madrid.

CEOE (2017). Línea abierta para la identificación de problemas de las empresas españolas en el Mercado único europeo, fase XIV: informe de los resultados del proyecto 2017, Madrid.

CNMC (2018). La CNMC analiza aspectos de las relaciones comerciales de las empresas de gas y electricidad con sus clientes (online). Recuperado el 13 de marzo de https://www.cnmc. es/2018-05-16-la-cnmc-analiza-aspectos-de-las-relacionescomerciales-de-las-empresas-de-gas-y

CNMV (2017). Actuación de la CNMV de supervisión de la comercialización de productos financieros en oficinas bancarias haciendo uso, por primera vez, del «cliente misterioso» o «mystery shopping», Madrid. Recuperado el 13 de marzo de https:// www.cnmv.es/loultimo/Comunicado\%20Mystery_shopping.pdf

Comisión Europea (2015). Legislar mejor para obtener mejores resultados - Un programa de la UE, Estrasburgo.

Comisión Europea (2016). Legal study on retail establishment through the 28 member states: Restrictions and freedom of establishment, Bruselas.

Comisión Europea (2017a). Completing the Better Regulation Agenda: Better solutions for better results, Estrasburgo.

Comisión Europea (2017b). Final Report. Single Market Forum: Generating Further Momentum for the Internal Market, Malta.

Comisión Europea (2018a). The Single Market in a changing world. A unique asset in need of renewed political commitment, Bruselas.

Comisión Europea (2018b). Report from the Commission on the application in 2017 of Regulation (EC) No 1049/2001 regarding public access to European Parliament, Council and Commission documents, Bruselas. Recuperado el 11 de marzo de 2019 
de https://ec.europa.eu/info/sites/info/files/com_2018_663_f1_report_from_commission_en_v3_p1_988979.pdf

Comisión Europea (2018c). Study to monitor the business and regulatory environment affecting the collaborative economy in the EU - final report, Bruselas.

Comision Europea (2018d). Study on the assessment of the regulatory aspects affecting the collaborative economy in the tourism accommodation sector in the 28 Member States, Bruselas.

Comisión Europea (2019). Register of Commission Expert Groups (online). Recuperado el 28 de marzo de 2019 de http:// ec.europa.eu/transparency/regexpert/index.cfm?do=groupDetail.groupDetail\&grouplD=1908

Comité Económico y Social (2016). Dictamen del Comité Económico y Social Europeo sobre «Normas preparadas para el futuro», Bruselas. Recuperado el 29 de marzo de 2019 de https://eur-lex.europa.eu/legal-content/ES/TXT/PDF/?uri=CELEX:52016AE2976\&from=DA

Consejo de Competitividad (2019). Revised draft Council Conclusions on an updated approach for a competitive Single Market -Borrador-ST 73572019 INIT. Recuperado el 29 de marzo de 2019 de https://data.consilium.europa.eu/doc/document/ ST-7691-2019-INIT/en/pdf

Díaz Mesanza, G. y Medina Asensio, P. (2017). Notificación de Normas y Reglamentaciones Técnicas. Revista Economía Industrial: Economía Experimental, (403), 155-159. Recuperado el 11 de marzo de 2019 de https://www.mincotur.gob.es/Publicaciones/Publicacionesperiodicas/Economialndustrial/RevistaEconomialndustrial/403/NOTA.pdf
Gobierno de Reino Unido (2017). Mystery Shopper results October-December 2017, Londres.

INAP (2015). Análisis de las Conferencias Sectoriales (2001-2012): Valores y Percepciones de los Agentes Políticos y Técnicos y Dinámica de Funcionamiento, Madrid.

Ministerio de Política Territorial y Función Pública (2018). Informe de seguimiento de la actividad de los ministerios en relación con los programas del marco general para la mejora de la calidad de la Administración General del Estado durante 2017, Madrid. Recuperado el 11 de marzo de http://www.mptfp.gob. es/dam/es/portal/funcionpublica/gobernanza-publica/calidad/ informes/ISAM/Informe_ISAM_2017.pdf.pdf

Ministerio de la Presidencia y para las Administraciones Territoriales (2016). Informe sobre la actividad de las conferencias sectoriales en 2016, MPAT, Madrid. Recuperado el 11 de marzo de 2019 de http://www.mptfp.gob.es/dam/es/ portal/areas/politica_autonomica/coop_autonomica/Conf_Sectoriales/Documentacion/Conf_Sect_anuales/parrafo/0/text_ es_files/INFORME_CC_SS_2016.pdf.pdf

OCDE (2015a). OECD Regulatory Policy Outlook 2015, París. OECD Publishing. Recuperado el 29 de marzo de 2019 de http://www.oecd.org/publications/oecd-regulatory-policy-outlook-2015-9789264238770-en.htm

OCDE (2015b). Key findings from the hearing discussion held during the 1st, 2nd, 3rd meeting of the OECD Competition Commitee on 16-18 June 2015, París.

Parlamento Europeo (2017). Mapping the Cost of Non-Europe, 2014-2019 - Fourth edition, Bruselas. European Parliamentary Research Service. 